\title{
AN EFFICIENT SYNTHESIS OF DUTASTERIDE: UTILIZING BENZOYL GROUP AS NOVEL LACTAMIC PROTECTING GROUP
}

\author{
Sachin B. Sawant ${ }^{1,2, *}$, Kalyan Chakravarthy Eadara ${ }^{1}$, Padi Pratap Reddy ${ }^{1}$, \\ Rajendra Agarwal ${ }^{1}$, and Renu Sharma ${ }^{2}$ \\ ${ }^{1}$ Department of Chemical Research \& Development, Macleods Pharmaceuticals Ltd, \\ Unit-V, Plot No.2209, GIDC Sarigam-396155, Gujarat, India \\ ${ }^{2}$ Department of Chemistry, NIMS University, Shobha Nager, Delhi-Highway, \\ Jaipur-303121, Rajasthan, India \\ *E-mail: sachin.sawant204@gmail.com
}

ABSTRACT

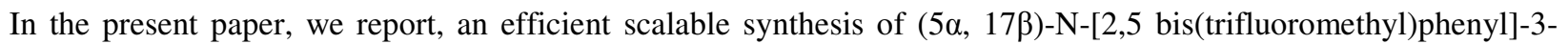

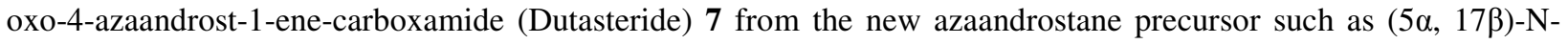
[2,5 bis(trifluoromethyl)phenyl]-3-oxo-4-benzoyl-4-aza-androstane $\quad$ carboxamide $\quad \mathbf{5}, \quad(5 \alpha, \quad 17 \beta)-\mathrm{N}-[2,5$ bis(trifluoromethyl)phenyl]-3-oxo-4-benzoyl-4-aza-androst-1-ene-carboxamide $\mathbf{6}$. The key strategic stage involve is benzoyl group act as a novel protecting group for lactamic NH group. The process was implemented for large scale for commercial launch.

Keywords: Dutasteride, Hyper-androgenic, N-benzoyldihydrodutasteride derivatives, Benzoyl group, Characterization.

(c) RASĀYAN. All rights reserved

\section{INTRODUCTION}

Dutasteride belongs to azasteriod class of compounds and function as a $5 \alpha$-reductase inhibitor ${ }^{1}$ which prevents the conversion of the androgen sex hormone testosterone into the more potent metabolite dihydrotestosterone (DHT). In 2009, South Korea has been licensed dutasteride for the treatment of androgenetic alopecia and in Japan 2015.<smiles>C[C@]12C=CC(=O)N[C@@H]1CCC1[C@@H]2CC[C@@]2(C)[C@@H](C(=O)Nc3cc(C(F)(F)F)ccc3C(F)(F)F)CC[C@@H]12</smiles>

7

Dutasteride used in the treatment of hyper androgenic conditions such as acne, ${ }^{2}$ hirsutism ${ }^{3}$ and benign prostate hypertrophy. Many synthetic methods have been reported for dutasteride 7 preparation. ${ }^{4-12}$ In the preparation of dutasteride, the introduction of the carbon-carbon double bond in conjugation with C-3 carbonyl carbon of azaandrosteriods is one of the most important chemical reaction. This dehydrogenation reaction could be achieved using the conventional reagents such as benzene-seleninic anhydride, 2,2 pyridyl disulfide and sodium metaperiodate, ${ }^{5} \mathrm{~N}, \mathrm{O}$-bis-(trimethylsilyl)trifluoroacetamide (BSTFA) and 2, 3-dichloro-5,6-dicyano-1,4-benzoquinone (DDQ), ${ }^{6}$ leads to unwanted toxic by-product such selenium compounds and some of reported pharmacopeia impurities such as $(5 \alpha, 17 \beta)-N-[2,5$ 


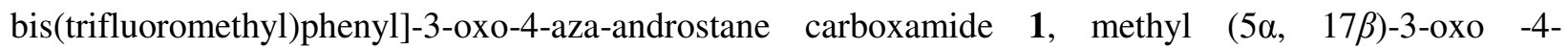
azaandrost -1-ene-17-carboxylate $2(5 \alpha, 17 \beta)-N$ - $N$-dimethyl-3-oxo-4-azaandrost-1-ene-17- carboxamide 3,(17 $\beta$ )- $N$-[2,5-Bis(trifluoromethyl)phenyl]-3-oxo-4- azaandrost-1,5(6)-diene-17 carboxamide 4 (Fig.-1) and thus affords poor quality of dutasteride drug substances. Literature reveals that dutasteride couldn't be easily purified using a conventional method such as recrystallization where it is mixed with impurities.

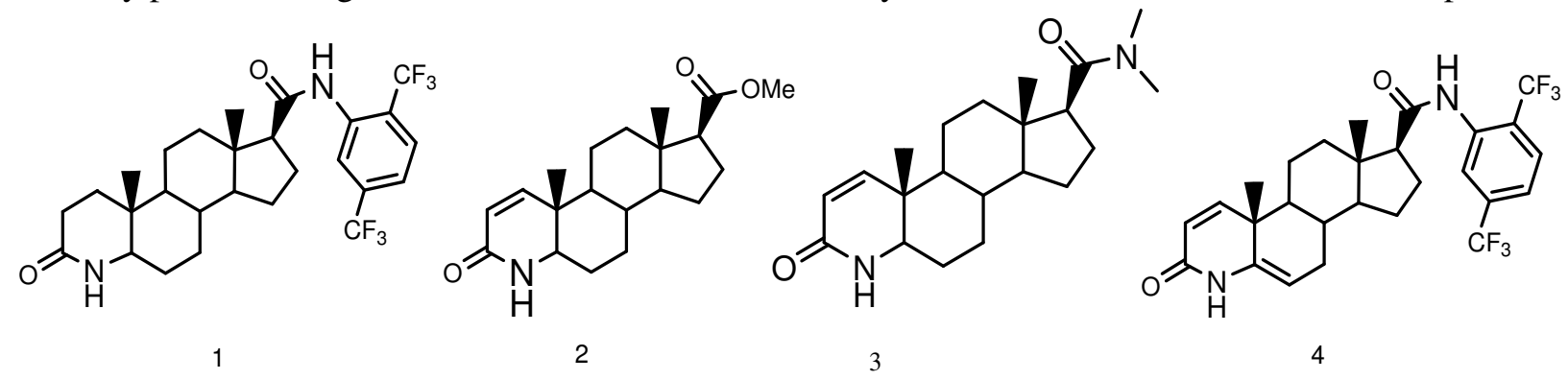

Fig.-1

It is an important to explore an efficient synthesis route that could yield highly pure dutasteride without involving any lengthy purification. The present work (Scheme-1) describes an industrially viable and improved process for the preparation of highly pure dutasteride from the novel intermediates. The merits of the present work are:

(1) The dehydrogenation reaction is executed in the penultimate stage also the resulting intermediates can be purified by crystallization from methanol and

(2) The de-protection of benzoyl group can be achieved using inexpensive reagents $70 \%$ aq. ethylamine and with mild reaction condition to afford pure dutasteride.

\section{General Procedure}

\section{EXPERIMENTAL}

All melting points were determined with polmon melting point apparatus ${ }^{1} \mathrm{H}-\mathrm{NMR}$ and ${ }^{13} \mathrm{C}-\mathrm{NMR}$ spectra were recorded on a Bruker $300 \mathrm{MHz}$ spectrometer. Chemical shifts are reported in ppm downfield from TMS as an internal standard. Mass spectra were measured on Perkin Elmer PE SCIEX-API 2000 mass spectrometer.

\section{Preparation of 5 a}

To a mixture of $(5 \alpha, 17 \beta)-N$-[2, 5 bis(trifluoromethyl)phenyl]-3-oxo-4-aza-androstane carboxamide1(100 $\mathrm{g}, 0.188$ moles) obtained by reported method. 4-dimethylaminopyridine (30 g, 0.24 moles), 4-Chloro benzoyl chloride $(45.0 \mathrm{~g}, 0.25$ and toluene $(1000 \mathrm{~mL})$ was refluxed for $5 \mathrm{~h}$. Then the mixture was evaporated under reduced pressure to obtain a crude residue. This was dissolved in methylene chloride $(700 \mathrm{~mL})$ and washed with water $(300 \mathrm{~mL})$. The methylene chloride layer was separated and concentrated under reduced pressure to obtain residue, which was treated with methanol $(400 \mathrm{~mL})$ to precipitate the product. The product was filtered, washed with water and dried to obtain 5a as a white powder $(107 \mathrm{~g}$, 85\%); mp 190-192 ${ }^{\circ} \mathrm{C} ;{ }^{1} \mathrm{H}$ NMR $\left(\mathrm{CDCl}_{3}, \delta \mathrm{ppm}\right): 0.79(\mathrm{~s}, 3 \mathrm{H}), 0.92-1.0(\mathrm{~m}, 2 \mathrm{H}), 1.19-1.24(\mathrm{~s}, 3 \mathrm{H}), 1.24(\mathrm{~s}$, $1 \mathrm{H}), 1.31-1.36(\mathrm{~m}, 2 \mathrm{H}), 1.39-1.47(\mathrm{~m}, 2 \mathrm{H}), 1.48-1.53(\mathrm{~m}, 2 \mathrm{H}), 1.71-1.73(\mathrm{~m}, 4 \mathrm{H}), 1.79-1.81(\mathrm{~m}, 1 \mathrm{H})$, 1.88-1.92 (m,1H), 2.02-2.09 (d, 1H), 2.25-2.34 (m, 1H), 2.37-2.47 (m, 1H), 2.49-2.60 (m, 1H), 2.63-2.66 (m, 1H), 7.39-7.45 (m, 3H- Ar-H), $7.54(\mathrm{~s}, 1 \mathrm{H}-\mathrm{NH}), 7.72-7.74(\mathrm{~d}, 1 \mathrm{H}, \mathrm{Ar}-\mathrm{H}), 7.78-7.80$ (d, 2H, -Ar-H), $8.76(\mathrm{~s}, 1 \mathrm{H},-\mathrm{ArH}) ;{ }^{13} \mathrm{C} \mathrm{NMR}\left(\mathrm{CDCl}_{3}, \delta \mathrm{ppm}\right): 12.8,13.45,21.12,23.69,29.52,30.11,32.80,34.76$, $36.04,37.91$, 44.71, 51.55, 58.39, 64.97, 120.38, 120.45, 121.54, 121.77, 121.84, 122.15, 124.49, 124.87, 126.76, 126.81, 126.86, 129.06, 130.75, 134.87, 135.20, 136.44, 139.87, 171.50, 172.99, 176.26. MS (ESI, m/z): 669.8

\section{Preparation of $\mathbf{5 b}$}

This compound was prepared in a similar way to 5a, using $\mathbf{1}$ (10g, 0.0188 moles), 4dimethylaminopyridine (3.0 g, 0.024 moles) and 4-nitrobenzoyl chloride (5.16g, 0.027 moles). The 
product $\mathbf{5 b}$ was obtained as a pale yellow solid (11.2g, 87\%); mp 244-246 ${ }^{\circ} \mathrm{C} ; ;{ }^{1} \mathrm{H} \mathrm{NMR}\left(\mathrm{CDCl}_{3}, \delta \mathrm{ppm}\right)$ : 0.81(s,3H, $\left.-\mathrm{CH}_{3}\right), 0.97-0.99(\mathrm{~m}, 1 \mathrm{H}),, 1.0-1.1(\mathrm{~m}, 1 \mathrm{H}), 1.23-1.27\left(\mathrm{~s}, 3 \mathrm{H},-\mathrm{CH}_{3}\right), 1.23-1.27(\mathrm{~s}, 1 \mathrm{H}), 1.32-1.41$ $(\mathrm{m}, 6 \mathrm{H}), 1.73-1.78(\mathrm{~m}, 3 \mathrm{H}), 1.91-2.0(\mathrm{~m}, 2 \mathrm{H}), 2.01-2.06(\mathrm{~m}, 1 \mathrm{H}), 2.11-2.13(\mathrm{~d}, 1 \mathrm{H}), 2.31-2.36(\mathrm{~m}, 1 \mathrm{H})$, 2.36-2.41 (t,1H), 2.49-2.65 (m, 1H), 2.65-2.68 (m, 1H), 3.71-3.72 (dd, 1H), 7.4 \& $7.94(\mathrm{~d}, 2 \mathrm{H}$, Ar$\mathrm{H}), 7.53(\mathrm{~s}, 1 \mathrm{H}, \mathrm{Ar}-\mathrm{H}), 7.92-7.94(\mathrm{~d}, 2 \mathrm{H}, \mathrm{Ar}-\mathrm{H}), 8.26-8.28$ (d, 2H, Ar-H), $8.77(\mathrm{~s}, 1 \mathrm{H},-\mathrm{NH}) ;{ }^{13} \mathrm{C}$ NMR $\left(\mathrm{CDCl}_{3}, \delta \mathrm{ppm}\right): 12.9,13.4\left(-\mathrm{CH}_{3}\right), 21.4,23.6,24.1,29.5,30.2,32.6,34.7,36.07,37.8,44.7,51.5,55.6$, 58.3, 65.2, 119.0, 120.4, 121.7, 122.1, 123.8, 124.8, 126.8, 127.1, 127.5, 129.7 134.8, 135.5, 136.4, 141.3, 150.0, 171.4, $173.5(-\mathrm{C}=\mathrm{O}), 175.5(-\mathrm{C}=\mathrm{O})$; MS (ESI, m/z): 680.3.



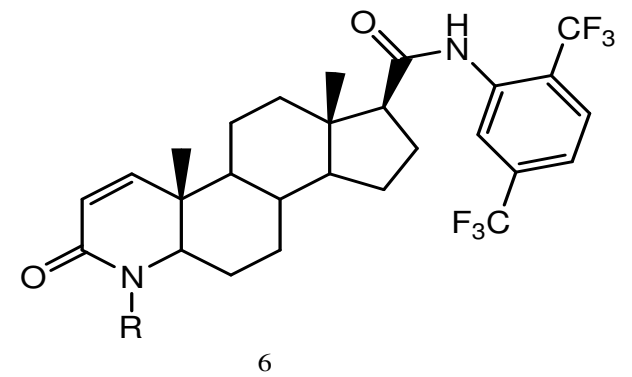

Compound 5 and $6 ; \mathrm{R}=$

a- $\quad p-\mathrm{Cl} \mathrm{C}_{6} \mathrm{H}_{4} \mathrm{CO}$

$c-\mathrm{NO}_{2} \mathrm{C}_{6} \mathrm{H}_{4} \mathrm{CO}$

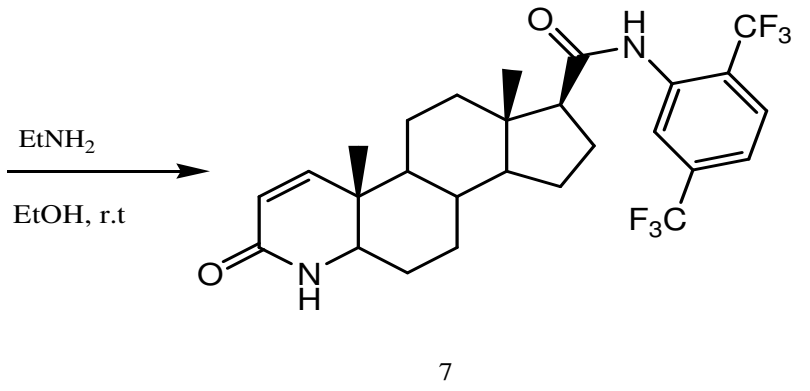

\section{Preparation of 5c}

This compound was prepared in a similar way to 5a, using $\mathbf{1}$ (10.0g, 0.0188 moles), 4dimethylaminopyridine (3.0 g, 0.024 moles) and 3-nitrobenzoyl chloride (5.16 g, 0.027 moles). The product $\mathbf{5 c}$ was obtained as a pale yellow solid $(10.8 \mathrm{~g}, 84 \%)$; mp $213-215{ }^{\circ} \mathrm{C} ;{ }^{1} \mathrm{H} \mathrm{NMR}\left(\mathrm{CDCl}_{3}, \delta \mathrm{ppm}\right)$ : $0.81\left(\mathrm{~s}, 3 \mathrm{H},-\mathrm{CH}_{3}\right), 0.97-0.99(\mathrm{~m}, 1 \mathrm{H}), 1.0-1.1(\mathrm{~m}, 1 \mathrm{H}), 1.23-1.27\left(\mathrm{~s}, 3 \mathrm{H},-\mathrm{CH}_{3}\right), 1.23-1.27(\mathrm{~s}, 1 \mathrm{H}), 1.32-$ $1.41(\mathrm{~m}, 6 \mathrm{H}), 1.73-1.78(\mathrm{~m}, 3 \mathrm{H}), 1.91-2.0(\mathrm{~m}, 2 \mathrm{H}), 2.01-2.06(\mathrm{~m}, 1 \mathrm{H}), 2.11-2.13(\mathrm{~d}, 1 \mathrm{H}), 2.31-2.36(\mathrm{~m}, 1 \mathrm{H})$, 2.36-2.41 (t, 1H), 2.49-2.65 (m, 1H), 2.65-2.68 (m, 1H),3.71-3.72 (dd, 1H) 7.4 \& $7.94(\mathrm{~d}, 2 \mathrm{H}$, Ar-H), 7.53 (s, 1H, Ar-H), 7.92-7.94 (d, 2H, Ar-H), 8.26-8.28 (d, 2H, Ar-H), 8.77(s ,1H, -NH) ; ${ }^{13} \mathrm{C}$ NMR $\left(\mathrm{CDCl}_{3}, \delta\right.$ ppm): 12.9, 13.4(- $\left.-\mathrm{CH}_{3}\right), 21.4,23.6,24.1,29.5,30.2$, 32.6, 34.7, 36.07, 37.8, 44.7, 51.5, 55.6, 58.3, $65.2,119.0,120.4,121.7,122.1,123.8,124.8,126.8,127.1,127.5,129.7134 .8,135.5,136.4,141.3$, 150.0, 171.4, 173.5(-C=O), 175.5(-C=O) ; MS (ESI, m/z): 680.3 .

\section{Preparation of 5d}

This compound was prepared in a similar way to 5a, using $\mathbf{1}$ (10.0 g, 0.0188 moles), 4dimethylaminopyridine (3.0 g, 0.024 moles) and benzoyl chloride $(5.0 \mathrm{~g}, 0.035$ moles $)$. The product 5d was obtained as a white solid $(9.7 \mathrm{~g}, 82 \%)$; mp 278-280 ${ }^{\circ} \mathrm{C} ;{ }^{1} \mathrm{H}$ NMR $\left.\left(\mathrm{CDCl}_{3}\right), \delta \mathrm{ppm}\right)$ : $0.71(\mathrm{~s}, 3 \mathrm{H}), 0.87-$ $0.97(\mathrm{~m}, 2 \mathrm{H}), 1.11-1.16(\mathrm{~s}, 3 \mathrm{H}), 1.22-1.25(\mathrm{~m}, 1 \mathrm{H}), 1.27-1.33(\mathrm{~m}, 2 \mathrm{H}), 1.35-1.41(\mathrm{~m}, 2 \mathrm{H}), 1.44-1.53(\mathrm{~m}$, 2H), 1.71-1.73 (m, 4H), 1.79-1.81 (m,1H), 1.88-1.92 (m,1H), 2.02-2.09 (d, 1H), 2.25-2.34 (m, 1H), 2.37$2.47(\mathrm{~m}, 1 \mathrm{H}), 2.49-2.60(\mathrm{~m}, 1 \mathrm{H}), 3.56-2.60(\mathrm{~d}, 1 \mathrm{H}), 7.33-7.35(\mathrm{~m}, 3 \mathrm{H}-\mathrm{Ar}-\mathrm{H}), 7.45-7.48(\mathrm{~d}, 2 \mathrm{H}-\mathrm{NH})$, 
RASĀYAN J. Chem.

Vol. 10 | No. 3 |997 - 1002 | July - September | 2017

7.63-7.65 (d, $1 \mathrm{H}, \mathrm{Ar}-\mathrm{H}), 7.78-7.80(\mathrm{~d}, 2 \mathrm{H},-\mathrm{Ar}-\mathrm{H}), 8.67$ (s, $1 \mathrm{H},-\mathrm{ArH}) ;{ }^{13} \mathrm{C} \mathrm{NMR}\left(\mathrm{CDCl}_{3}, \delta \mathrm{ppm}\right): 11.73$, $12.40,2.0 .0,22.63,23.15,28.51,29.0,31.86,33.74,35.0,36.89,43.69$, 50.53, 54.66, 57.35, 63.89, $119.37,119.4,120.53,120.73,120.83,121.11,123.45,123.83,125.71,125.76,125.81,126.16,127.66$, $128.4,132.45,133.83,134.16,134.65,135.41,170.48,171.74,176.20 . ;$ MS (ESI, m/z): 635.4 .

\section{General Procedure for the synthesis of compounds 6a-d Preparation of 6 a}

A mixture of benzoyl derivative 5a $(9.0 \mathrm{~g}, 13.46 \mathrm{mmol})$, DDQ (6.4 g, $28.19 \mathrm{mmol})$, bis (trimethylsilyl) trifluoroacetamide (BSTFA, $18.0 \mathrm{~g}, 70.04 \mathrm{mmol}), 3$ drops of triflic acid and xylene $(50 \mathrm{~mL})$ were stirred at ambient temperature for 1-2 hr. The reaction mixture was then stirred at mild reflux for $4 \mathrm{hr}$. The solvent was removed under reduced pressure, and the product was isolated from methanol to get $6 \mathbf{a}$ as a white solid $(8.1 \mathrm{~g}, 89 \%)$; mp 209-211 ${ }^{\circ} \mathrm{C} ;{ }^{1} \mathrm{H}$ NMR $\left(\mathrm{CDCl}_{3}\right)$ : $0.82(\mathrm{~s}, 3 \mathrm{H}), 1.12-1.25(\mathrm{~m}, 2 \mathrm{H}), 1.30$ (s, $3 \mathrm{H}), 1.36-1.38(\mathrm{~m}, 3 \mathrm{H}), 1.39-1.43(\mathrm{~m}, 3 \mathrm{H}), 1.59-1.76(\mathrm{~m}, 4 \mathrm{H}), 1.8-1.9(\mathrm{~m}, 3 \mathrm{H}), 2.26-2.28(\mathrm{t}, 1 \mathrm{H}), 3.71-3.74$ $(\mathrm{d}, 1 \mathrm{H}), 5.72-5.75(\mathrm{~d}, 1 \mathrm{H}), 6.91-6.93(\mathrm{~d}, 1 \mathrm{H}), 7.30-7.34(\mathrm{t}, 3 \mathrm{H}-\mathrm{Ar}-\mathrm{H}), 7.35-7.41(\mathrm{~d}, 1 \mathrm{H} \mathrm{Ar}-\mathrm{H}), 7.43(\mathrm{~s}$, 1H,-NH proton), 7.45-7.61 (d, 1H, Ar-H), 7.63-7.80 (d, 2H, Ar-H), 8.60 (s, 1H, Ar-H); ${ }^{13} \mathrm{C} \mathrm{NMR}\left(\mathrm{CDCl}_{3}\right.$, $\delta$ ppm): $120.29,120.46,121.26,121.70,121.78,122.54,124.88,125.32,126.67,126.82,126.89,128.49$, $128.89130 .92,134.35,134.46,135.33,136.42,139.70,152.4,166.4,171.26,174.3 ; \mathrm{MS}(\mathrm{ESI}, \mathrm{m} / \mathrm{z})$ : 667.8

\section{Preparation of $6 b$}

This compound was prepared in a similar way to $6 \mathbf{a}$, using compound $\mathbf{5 b}$ (9.0 g, $13.24 \mathrm{mmol})$, DDQ (4.85 $\mathrm{g}, 21.36 \mathrm{mmol})$, BSTFA $(15.42 \mathrm{~g}, 59.9 \mathrm{mmol})$ and 1 drop of triflic acid. The product $\mathbf{6} \mathbf{b}$ was obtained as a white solid $(7.5 \mathrm{~g}, 83 \%)$; mp 230-232 ${ }^{\circ} \mathrm{C} .{ }^{1} \mathrm{H}$ NMR $\left(\mathrm{CDCl}_{3}, \delta \mathrm{ppm}\right): 0.83(\mathrm{~s}, 3 \mathrm{H}), 1.1-1.19(\mathrm{~m}, 2 \mathrm{H}), 1.28$ $(\mathrm{s}, 4 \mathrm{H}), 1.33-1.78(\mathrm{~m}, 5 \mathrm{H}), 1.78-1.9(\mathrm{~m}, 2 \mathrm{H}), 1.79-1.94(\mathrm{~m}, 2 \mathrm{H}), 1.97-2.16(\mathrm{~d}, 1 \mathrm{H}), 2.19-2.30(\mathrm{~m}, 1 \mathrm{H})$, 2.37-2.42 (m, 2H), 3.85-3.88 (d, $\left.1 \mathrm{H}, \mathrm{C}_{2}-\mathrm{H}\right), 7.10-7.12\left(\mathrm{~d}, 1 \mathrm{H}, \mathrm{C}_{1} \mathrm{H}\right), 7.45-7.42(\mathrm{~d}, 1 \mathrm{H}, \mathrm{Ar}-\mathrm{H}), 7.53(\mathrm{~s}, 1 \mathrm{H}$, -NH), 7.73-7.75 (d, 1H, -Ar-H), 7.94-7.96 (d, 2H, -Ar-H), 8.26-8.28 (d, 2H, Ar-H), $8.77 \mathrm{~s}, 1 \mathrm{H},-\mathrm{Ar}-\mathrm{H})$; ${ }^{13} \mathrm{C}$ NMR $\left(\mathrm{CDCl}_{3}, \delta \mathrm{ppm}\right): 13.4\left(-\mathrm{CH}_{3}\right), 14.2,21.2,29.5,35.0,37.8,40.1,44.6,47.9,55.6,58.2$, $64.7,120.3,120.3,120.4,120.5,121.4,121.7,122.1,122.2,123.7,124.4,124.8,126.8,129.9,134.9$, 135.2, 136.3, 141.5, 149.9, 153.4, 166.5, 171.2, 173.5(C=O); MS (ESI, m/z): 678.2

\section{Preparation of 6c}

This compound was prepared in a similar way to $\mathbf{6 a}$, using compound $\mathbf{5 c}(9.0 \mathrm{~g}, 13.24 \mathrm{mmol})$, DDQ (4.85 $\mathrm{g}, 21.36 \mathrm{mmol})$, BSTFA $(15.42 \mathrm{~g}, 59.9 \mathrm{mmol})$ and 1 drop of triflic acid. The product $6 \mathbf{c}$ was obtained as a white solid $(7.8 \mathrm{~g}, 86 \%)$; $\mathrm{mp} 157-159^{\circ} \mathrm{C} .{ }^{1} \mathrm{H}$ NMR $\left(\mathrm{CDCl}_{3}, \delta \mathrm{ppm}\right): 0.82(\mathrm{~s}, 3 \mathrm{H}), 1.1-1.3(\mathrm{~m}, 3 \mathrm{H}), 1.3(\mathrm{~s}$, $3 \mathrm{H}), 1.36-1.42(\mathrm{~m}, 1 \mathrm{H}), 1.45-1.57(\mathrm{~m}, 4 \mathrm{H}), 1.77-1.85(\mathrm{~m}, 2 \mathrm{H}), 1.89-1.96(\mathrm{~m}, 2 \mathrm{H}), 2.16-2.19(\mathrm{~d}, 1 \mathrm{H}), 2.3-$ $2.43(\mathrm{~m}, 3 \mathrm{H}), 3.86-3.9(\mathrm{dd}, 1 \mathrm{H}), 5.85-5.88(\mathrm{~d}, 1 \mathrm{H},-\mathrm{H}), 7.1-7.13\left(\mathrm{~d}, 1 \mathrm{H}, \mathrm{C}_{1}-\mathrm{H}\right)$, 7.45-7.47 (d, $\left.1 \mathrm{H}, \mathrm{Ar}-\mathrm{H}\right)$, 7.54 (s, 1H,- NH), 7.62-7.66 (t, 1H, Ar-H), 7.73-7.75 (d, 1H, -Ar-H), 8.13-8.15 (d, 1H, Ar-H), 8.37-8.39 (dd, 1H, Ar-H), 8.63-8.64 (t,1H, Ar-H), $8.76(\mathrm{~s}, 1 \mathrm{H}, \mathrm{Ar}-\mathrm{H}) ;{ }^{13} \mathrm{C} \mathrm{NMR}\left(\mathrm{CDCl}_{3}, \delta \mathrm{ppm}\right): 13.4,14.2,21.2$, 29.5, 35.0, 37.8, 40.1, 44.7, 47.9, 55.6, 58.2, 64.6, 120.3,120.4, 120.5, 121.1, 121.5, 122.1, 122.2, $124.1,124.4,124.8,126.8,127.1,129.7,134.7,135.2,136.3,137.8,148.2,153.4,166.6,171.3,173.2$ $(\mathrm{C}=\mathrm{O}) ; \mathrm{MS}(\mathrm{ESI}, \mathrm{m} / \mathrm{z}): 678.2$

\section{Preparation of $6 d$}

This compound was prepared in a similar way to $\mathbf{6 a}$, using compound $\mathbf{5 d}(9.0 \mathrm{~g}, 14.18 \mathrm{mmol})$, DDQ (5.10 g, $22.46 \mathrm{mmol})$, BSTFA (16.0 g, $62.25 \mathrm{mmol})$ and 1 drop of triflic acid. The product $\mathbf{6 d}$ was obtained as a white solid (8.3 g, $92 \%)$; mp 269-271 ${ }^{\circ} \mathrm{C} ;{ }^{1} \mathrm{H}$ NMR $\left(\mathrm{CDCl}_{3}, \delta \mathrm{ppm}\right): \delta 0.69(\mathrm{~s}, 3 \mathrm{H}), 0.94-1.04(\mathrm{~m}, 2 \mathrm{H})$, $1.30(\mathrm{~s}, 3 \mathrm{H}), 1.36-1.38(\mathrm{~m}, 3 \mathrm{H}), 1.39-1.43(\mathrm{~m}, 3 \mathrm{H}), 1.59-1.76(\mathrm{~m}, 4 \mathrm{H}), 1.8-1.9(\mathrm{~m}, 3 \mathrm{H}), 2.25-2.30(\mathrm{~m}$, $1 \mathrm{H}), 3.85-3.86(\mathrm{~d}, 1 \mathrm{H})$, 5.85-5.88 (d, 1H), 7.02-7.06 (d, 1H- Ar-H), $7.26(\mathrm{~s}, 2 \mathrm{H} \mathrm{Ar}-\mathrm{H}), 7.47$-7.75 (d, 1H, Ar-H), 7.80-7.83 (d, 2H, Ar-H), 8.77- 8.79 (s, $1 \mathrm{H}, \mathrm{Ar}-\mathrm{H}) ;{ }^{13} \mathrm{C}$ NMR $\left(\mathrm{CDCl}_{3}, \delta \mathrm{ppm}\right): 12.43,12.95,20.18$, 22.36, 22.68, 23.08, 28.45, 33.99, 36.70, 38.97, 43.67, 46.91, 54.60, 57.01, 63.25, 119.55, 119.78, 119.82, 
$120.76,120.99$, 121.0, 121.29, 121.49, 123.47, 123.78, 125.82, 125.88, 127.59, 128.58, 132.33, 133.69, 134.0, 134.8, 135.4, 151.34, 165.41, 170.51, 174.4, MS (ESI, m/z): Found: 633.39

\section{General Procedure for the de-protection}

A mixture of $6 \mathrm{a}-\mathrm{d}(8.0 \mathrm{~g}, 15.0 \mathrm{mmol}), 70 \%$ aqueous ethylamine solution $(1.5 \mathrm{~mL}, 23.33 \mathrm{mmol})$ and ethanol $(50 \mathrm{~mL})$ were stirred at ambient temperature for $15 \mathrm{hr}$. The solvent was removed under reduced pressure and the product was isolated in a mixture of ethyl acetate and tetrahydrofuran to obtain $\mathbf{7}$ as a white solid. This product was suspended in water $(500 \mathrm{~mL})$ and stirred at room temperature for $4 \mathrm{~h}$. The product was filtered and dried to get dutaseride ( $4.8 \mathrm{~g}, 88 \%$ ); mp $250-251{ }^{\circ} \mathrm{C} .{ }^{1} \mathrm{H} \mathrm{NMR},{ }^{13} \mathrm{C} \mathrm{NMR}$, and Mass data have been described in the text. Thus obtained 7 is high in pure i.e. $99.9 \%$ with $\leq 0.05 \%$ of impurity $1,0.01 \%$ of impurity $2,0.05 \%$ of impurity $3,0.02 \%$ of impurity 4 by HPLC.

\section{RESULTS AND DISCUSSION}

Initially, $(5 \alpha, 17 \beta)-N$-[2,5 bis(trifluoromethyl)phenyl]-3-oxo-4-aza-androstane carboxamide $\mathbf{1}$ was treated with benzoyl chloride or substituted benzoyl chloride in the presence of 4-dimethylaminopyridine to obtain $N$-benzoyldihydrodutasteride derivatives (5a-d) (Scheme-1) The structures of $\mathrm{N}$ benzoyldihydrodutasteride derivatives were characterized by ${ }^{1} \mathrm{H}-\mathrm{NMR},{ }^{13} \mathrm{C}-\mathrm{NMR}$, and mass spectral data. The silylation of amide functionality of $\mathrm{N}$-benzoyldihydrodutasteride using N,O-bis(trimethylsilyl)trifluoroacetamide (BSTFA) leads to formation of enolization towards the $\Delta^{2}$ position favours the C-2 carbon of the silylated compound to react with 2,3-dichloro-5,6-dicyano-1,4-benzoquinone (DDQ) to yield intermediate complex, which decomposed due to thermal treatment favour the dehydrogenation at the $\Delta^{1,2}$ position and thus affords the $N$-benzoyl dutasteride derivatives (6a-d). The isolation of pure $N$ benzoyl dutasteride derivatives (6a-d) obtained from its crystallization from methanol. It is observed that dutasteride related impurities including pharmacoepial impurities (1-4) get eliminated in the methanol filtrate.

In the present work, dutasteride was obtained after deprotecting the lactam N-benzoyl group of compounds (6a-d), provides a novel method for protection and deprotection of lactam NH group of azaandrostane derivatives. The hydrolysis of benzoyl group with hydrazine hydrate gives excellent yield dutasteride with 98\% purity; however, the de-protection carried out with aqueous ethylamine solution in ethanol gives advantageously desired product. Finally, crude dutasteride product on crystallization from the acetonitrile yielded highly $99.8 \%$ pure dutasteride (7a-d) as seen by HPLC method.

In conclusion, we have developed a concise, robust process for the production of dutasteride. The synthesis features provide a new industrially viable process to prepare dutasteride with more than $99.5 \%$ purity by the simple and convenient process. The process involves the protection and deprotection of benzoyl group on the lactam NH group of azaandrostane derivatives and precise control over the purity of final drug substances.

\section{ACKNOWLEDGEMENT}

Authors thank the management of Macleods Pharmaceuticals Limited, Mumbai for their support. Authors also thanks to the Analytical Research Department for their help in this work.

\section{REFERENCES}

1. G.H. Rasmusson, G.F. Reynolds, N.G. Steinberg, E .Walton, G.F. Patel, T. Liang, M.A. Cascieri. A.H. Cheung, J.R. Brooks and C. Berman, J. Med. Chem., 29, 2298 (1986).

2. L. S. Smith and J. J. Tegeler, Ann. Rep. Med. Chem., 24, 177 (1989).

3. J. R. Book, Clin. Endocrinol. Metab., 15, 391 (1986).

4. D. V. N. S. Rao, N. T. Ganala, J. P. Koilpillai, D. Ramesh, S. Meenaskshisundaram and N Andra, J. Hetrocyclic Chem., 44, 663 (2007).

5. M. Jasic, H. Kollmann, B. Lachmann, C. Noe, K. Zobl, WO Patent 066,195 (2005).

6. A. Bhattacharya, H. Dimicheleulf, W. Dollingalan, J. Douglas Edward, J. Grabowski, J. Am. Chem. Soc., 110, 3318 (1988). 
RASĀYAN $J$. Chem.

Vol. 10 | No. 3 |997 - 1002 | July - September | 2017

7. X. Peng, Y. Zheng-yu, Z. X. Hao-bing, Z. Ke-hua, S. Xun, C. Ying and Z. Yun-quing, Hetrocycles, 47, 703 (1998).

8. A. Bhattacharya, J. Williams, J. S. Amato, J.U.-H. Doling and E. J. Grabowski, Synth. Commun., 30, 2683 (1990).

9. N. Schaerer, B Weber, B W. Mueller. WO Patent 007, 523 (2004).

10. Gorgojo, J.M. Lobato, B.L. Antonio Lorente, J. Martin Juarez, WO Patent 029, 267 (2003).

11. Y.H. Moon, D.J. Kim, C-H. Park, K. I. Lee, J. C. Lee, G S. Lee, WO Patent 007,670, (2005).

12. K. Satyanarayan, C. N. Rao, V. Himabindu and G. M. Reddy, Rasayan J. Chem., 2, 322(2008)

[RJC-1815/2017] 